\title{
BMJ Open UK advanced practice nurses' experiences of the COVID-19 pandemic: a mixed-methods cross-sectional study
}

\author{
Emily Wood (D), Rachel King, Michaela Senek, Steve Robertson, \\ Bethany Taylor (D) , Angela Tod (D) , Anthony Ryan
}

To cite: Wood E, King R, Senek M, et al. UK advanced practice nurses' experiences of the COVID-19 pandemic: a mixed-methods crosssectional study. BMJ Open 2021;11:e044139. doi:10.1136/ bmjopen-2020-044139

- Prepublication history is published online only. To view please visit the journal online (http://dx.doi.org/10.1136/ bmjopen-2020-044139).

Received 25 August 2020 Revised 24 February 2021 Accepted 05 March 2021

\section{Check for updates}

(c) Author(s) (or their employer(s)) 2021. Re-use permitted under CC BY-NC. No commercial re-use. See rights and permissions. Published by BMJ.

Health Sciences School, The University of Sheffield, Sheffield, UK

Correspondence to

Dr Emily Wood;

e.f.wood@sheffield.ac.uk

\section{ABSTRACT}

Objective The aim of the study was to understand the experiences of advanced practice nurses (APNs) in the UK during the 2020 COVID-19 pandemic, particularly in relation to safety, shortages and retention.

Design A cross-sectional, mixed-methods survey.

Setting APNs in any UK setting.

Participants The survey was sent to an existing UK-wide cohort of APNs. 124 APNs responded (51\%).

Results UK-based APNs in this study reported shortages of staff (51\%) and personal protective equipment (PPE) $(68 \%)$ during the first 3 months of the coronavirus outbreak. Almost half (47\%) had considered leaving their job over the same 3 months. Despite difficulties, there were reports of positive changes to working practice that have enhanced care.

Conclusion UK APNs report COVID-19-related shortages in staff and equipment across primary and secondary care and all regions of the UK. Shortages of PPE during a pandemic are known to be a factor in the development of mental health sequelae as well as a risk factor for increased turnover and retention issues. Half of APNs surveyed were considering a change in job. The UK risks a further crisis in staff morale and retention if this is not acknowledged and addressed. APNs also expressed concern about patients not receiving routine care as many specialties closed or reduced working during the crisis. However, there were also many examples of good practice, positive changes and innovation.

\section{BACKGROUND}

The effect of COVID-19 on the healthcare workforce is a global concern. The coronavirus SARS-CoV-2 causes the respiratory disease COVID-19. ${ }^{1}$ Although it emerged in late 2019 in China, the first confirmed case of transmission in the UK was on 28 February $2020 .^{2}$ The UK's first case of the disease occurred several weeks after other European countries including Italy, France, Germany and Spain. ${ }^{2}$ Therefore, the UK government had advanced warning about the potential scale of the crisis and some preparation measures were put in place. For example, stopping all routine surgery, ${ }^{3}$ expanding inpatient capacity with the building of temporary Nightingale hospitals ${ }^{4}$

\section{Strengths and limitations of this study}

- The study examines the experiences of advanced practice nurses (APNs) in the National Health Service at the height of the COVID-19 crisis, including but not limited to, safety, personal protective equipment shortages and innovative practice.

- The use of an existing cohort of APNs allowed for access to a key healthcare group at the height of the crisis.

- The survey was only open for 2 weeks and the target group were very busy during this time, which affected our response rate.

- Although nurses from all four nations were included, Scotland and Northern Ireland were under-represented.

and opening a temporary professional register to enable recently retired healthcare staff to return to work. ${ }^{5}$ Despite this, there have been reports in medical journals, newspapers and on healthcare professionals' social media forums that healthcare staff were not adequately prepared and lacked access to key equipment and training for the new role, and that this may have contributed to loss of life. ${ }^{6}$

In crisis situations, it is common to redeploy staff to specialties that experience an increased workload or staffing shortages. This type of redeployment is included in most major incident planning. However, a lack of training for such new roles has been one concern linked to keeping healthcare staff safe. ${ }^{7}$ For instance, previous studies have found that the prospect of moral injury to staff who have to make challenging decisions in the crisis is high. ${ }^{8}$ In China, the effect of redeployment to infectious disease units led to staff feeling fear, exhaustion and powerlessness. ${ }^{9}$ To mitigate the adverse effect of the pandemic on mental health, in the UK, the British Psychological Society has released guidance to ensure sustained staff well-being during the crisis. ${ }^{10}$ 
Concerns about the lack of access to supply and training for use of personal protective equipment (PPE) have been reported in parts of the $\mathrm{UK}^{11}$ and elsewhere. A recent Royal College of Nursing survey of nurses found that $40 \%$ of nurses working in high-risk areas were concerned about the amount of PPE available. ${ }^{12}$ In Iran, access to PPE predicted lower distress, better physical health and greater job satisfaction, ${ }^{13}$ thus suggesting PPE protects the health worker's mental health as well as their physical health, so a lack of PPE in the UK is a concern in an already overstretched workforce. Taiwan's lessons learnt from their SARS outbreak led to the implementation of a strict system of zoning for patients and healthcare professionals alike, with the aim of improving the confidence of health workers so they continued to work through the crisis and stayed well. ${ }^{14}$ This strategy has been used in the UK, in the creation of hot hubs in primary care, COVID-19 wards in hospitals and altering treatment pathways to minimise the potential exposure of, for example, patients with cancer to SARS-CoV-2. ${ }^{15}$

This study focuses on the experiences of advanced practice nurses (APNs) during the COVID-19 pandemic. Advanced nursing roles such as APNs have increased across the world. ${ }^{16}$ In the UK, advanced level nursing roles should incorporate the four pillars of advanced practice: clinical practice, teaching, leadership and research. ${ }^{17}$ As experienced clinicians, APNs have a key role in responding to a health emergency such as COVID19. This is especially true where APNs work in frontline or gatekeeping services such as intensive treatment units, primary care and the emergency department. New international APN guidelines were published in May 2020, during the pandemic. The timing was coincidental but extremely pertinent. APNs as nurse leaders means they also have a 'role to play in prevention and containment of disease as well as providing care ${ }^{18}{ }^{18}$ APNs in the UK are highly trained, experienced healthcare professionals but even so, if redeployed to an unfamiliar area or not provided with the PPE or training, they could be at risk of both COVID-19 and the psychological and moral injuries arising from fear and distress. This study used an existing cohort of APNs from across the UK to study the experiences of advanced nurses during the initial stages of the outbreak and the impact of COVID-19 on the role of the $\mathrm{APN}$ and service delivery.

\section{DESIGN}

A cross-sectional mixed-methods survey of APNs in the UK.

\section{METHOD}

\section{Sample/participants}

An online survey was sent to all 243 members of an existing research cohort of APNs ${ }^{19}$ between 7 and 14 June 2020. The cohort consisted of APNs with a range of job titles and seniority within the National Health Service
(NHS) across primary and secondary care and covered all four nations of the UK. All had previously consented to join the cohort and be sent questionnaires throughout the 5-year project. The survey was open for 2 weeks and participants were entered into a prize draw to win high street vouchers.

\section{Survey design}

There were 49 questions on the survey, it contained both closed questions and open questions (eight questions) with free-text extended responses. Open-ended questions were included to enable us to explore nurses' experiences and how they had been affected rather than just if they had or not. Questions covered the preparedness of the participants' organisation at the start of the outbreak. It included questions about impact on patient and staff safety, shortages of staff and equipment, concerns, ability to access guidelines and advice, policy regarding staff sickness and demographics (six questions: setting, UK region, age, ethnic background, gender and disability). Topics were chosen based on contemporary anecdotal reports from healthcare professionals in the UK about the issues that COVID-19 had caused in the NHS.

The survey was piloted by APNs $(n=3)$ working in clinical practice. This ensured that the technology used for data collection was easily accessible to participants and that the questions were comprehensible, lacking ambiguity or repetition.

The survey was compiled using Google forms and sent to the email address provided by the cohort participants. Most questions required an answer to limit the amount of missing data. The only exceptions to this were where questions followed up from previous answers (for example, if you answered yes to the previous question, please give examples).

\section{Quantitative analysis}

Analysis of the overall preparedness used descriptive statistics in SPSS V.25. We explored the data to identify distribution of responses, trends and outliers. Data were first analysed with descriptive statistics using proportions for categorical variables. Analysis of the survey was primarily descriptive to discover differences between settings, geographical locations to compare how prepared organisations were and what issues arose as the pandemic progressed. Correlation was assessed using Spearman's rho test. Correlational analysis looked for relationships in key areas, such as perceived safety, access to PPE, ability to raise concerns and intention to leave as well as geographical differences across the UK. A probability below 0.05 $(p<0.05)$ was considered to be a significant difference.

\section{Qualitative analysis}

The free-text responses were analysed using Framework. ${ }^{20}$ The framework analysis involved five steps: familiarisation, identifying a thematic framework, indexing, charting, and mapping and interpretation. 
The Quirkos software package V.2.3.1 was used to help with data handling. The cloud version enabled three researchers (RK, TR, BT) to analyse the qualitative data. The Framework use began with themes of 'staff safety' and 'patient safety'.

\section{Integration}

Integration was done primarily at two stages: planning and interpretation. In the planning stage, open-ended questions were included to follow up where we expected nurses may be able to reveal more about their experiences. In the interpretation stage, we looked for significant or interesting results from the quantitative analysis to see if these could be explained in the qualitative data. This was done primarily by EW, RK and TR.

\section{Patient and public involvement}

APN stakeholders were involved in the initial design of the cohort and continue to be involved in the cohort research. This particular survey did not directly involve stakeholder or patient and public involvement in the development of the research questions, however the research team agreed these based on multiple anecdotal reports of issues facing nurses and other healthcare professionals on social media, mainstream media and medical journals. APN practitioners are involved in dissemination planning.

\section{RESULTS}

In total, $124 \mathrm{APNs}$ completed the survey, a response rate of $51 \%$ (see table 1 for details of the participants). Reasons for non-response were not collected. All four nations of the UK were represented.

Table 1 Demographics of respondents by setting, region and demographics

\begin{tabular}{lll}
\hline & & $\begin{array}{l}\text { Percentage of } \\
\text { respondents }\end{array}$ \\
\hline $\begin{array}{l}\text { Primary/secondary } \\
\text { care }\end{array}$ & $\begin{array}{l}\text { Primary care } \\
\text { Secondary care }\end{array}$ & $57(n=71)$ \\
& $36(n=45)$ \\
Region & Scoth & $7(n=8)$ \\
& Wales & $5(n=6)$ \\
& Northern Ireland & $7(n=8)$ \\
& England $(n=2)$ \\
Gender & Female & $86(n=108)$ \\
Age & Under 40 & $86(n=106)$ \\
& $41-50$ & $18(n=22)$ \\
& Over 50 & $40(n=50)$ \\
Ethnic background & White British & $92(n=52)$ \\
Disability or long-term & No health condition & $74(n=92)$ \\
health condition & & \\
\hline
\end{tabular}

English regions: $19 \%(n=23)$ Yorkshire and North East, 9\% $(n=11)$ North West, $5 \%(n=6)$ West Midlands, 17\% $(n=21)$ East Midlands and East, 23\% $(n=28)$ South East, 15\% $(n=19)$ South West.

\section{Quantitative findings}

Of concern is that $39 \%(n=48)$ thought their risk of exposure to the coronavirus could have been reduced and $21 \%$ $(n=26)$ did not feel their safety was prioritised during the crisis, this rose to $27 \%$ of APNs working in secondary care settings. Shortages of PPE were reported by $69 \% \quad(n=85)$ and $51 \%(n=63)$ reported shortages of staff.

Forty-one per cent of staff were redeployed $(n=51)$ and $22 \%(\mathrm{n}=27)$ had been asked to supervise others who were redeployed to their area. Forty-three per cent of staff $(n=54)$ reported working more overtime than usual and for $23 \% \quad(n=29)$ of respondents some of this was unpaid.

Over the previous 3 months (April-June 2020), 43\% $(n=53)$ of respondents had considered leaving their job. This included $25 \%$ of respondents $(n=29)$ who were considering leaving nursing completely. Twenty-two per cent of respondents $(n=27)$ reported being more likely to leave their job now, than before the pandemic; conversely, another 22\% $(\mathrm{n}=27)$ reported being less likely to leave, with $56 \%(\mathrm{n}=70)$ reporting no change in intention to leave since before the crisis.

We explored the potential reasons for APN's intention to leave. There was a significant correlation between intention to leave their current role and not being able to provide the same standard of care as they did before the crisis $(n=124$, correlation statistic $=-0.218, p=0.015)$, and between intent to leave and not feeling their safety was prioritised $(n=124$, correlation statistic $=-0.348, p=0.01)$. There were significant correlations between increased intention to leave and not feeling there was sufficient communication from management about coronavirus planning $(\mathrm{n}=124$, correlation statistic $=-0.195, \mathrm{p}=0.03)$.

There were no significant correlations between demographic characteristics (setting, region, gender, age, ethnic background or disability) and intention to leave, safety concerns or workload changes. However, this could be due to small numbers of representatives in some of the characteristics rather than there genuinely being no difference.

\section{Qualitative findings}

Open-ended questions were asked about participants' main concerns at work and their research priorities for the future. Key themes from this data were safety concerns, both for themselves and for their families, what worked well and research priorities.

\section{Safety concerns}

APNs were clearly concerned about protecting their own health, and that of their family members.

Stress from colleagues and public about COVID especially as three of my colleagues died from COVID symptoms. (APN 49)

The stress and psychological effect on my mental health, [of worrying about] taking COVID home to my partner. (APN 63) 
This concern was compounded by their experiences of inadequate PPE provision; with the expectation from employers to limit its use.

We were told that there was a shortage of PPE a couple of weeks into the pandemic, so we were asked not to wear it for everyone, so it didn't run out. (APN 75)

PPE was very poor quality, the gowns were made of tissue paper and the sleeves fell off, visors were flimsy and not suitable for reuse, but this was expected. I did not blame the employer, PHE national guidance was shameful. (APN 95)

They revealed concerns about delayed referrals and investigations, care home outbreaks and the risks associated with remote working.

We were responsible for assessing clinical condition and deciding treatment over the phone with literally no clinical information (temperature, heart rate, respiratory rate, oxygen saturation) when we know COVID causes severe hypoxia which can be asymptomatic. Clinical guidelines were available but useless without the tools for assessment. (APN 95)

\section{What has worked well?}

Some APNs described aspects of their work that had been effective in managing the challenges related to the pandemic. These included video consultations, utilisation of advanced roles (for example, advanced clinical practitioners, physician's associates and junior doctors being placed on the same rota). They also recognised the benefit of being able to give more time to seriously unwell patients (because there are less patients overall).

I am extremely proud at how well our practice has developed new and effective ways of working and all done very quickly, we had cameras for video consults within the first weeks of March. We put Infection Prevention and Control systems in place to screen patients and also looked at innovative ways of keeping our staff and patients safe with accessing PPE/visors etc. General Practice in general should be commended on how quickly they responded to the outbreak. (APN 29)

Feel standard [of care is] as good if not better. Lower contacts mean more thorough and more time for patients with more serious complaints. (APN 13)

This was not the case in all services, but many did report that patient contacts were much lower than usual; this allowed them to spend more time with each patient as they were not under as much time pressure as usual. Some services closed to all but emergency cases, others found that potential patients stayed away.

\section{Research priorities}

A wide range of ideas were put forward as suggestions for future COVID-19-related research. There was clearly a desire to understand the best ways to support the mental health of both staff and patients.

How to support nurses who have gone through the trauma of caring for COVID patients. (APN 64)

Similarly, it was recognised that many patients will require support in rehabilitating following COVID-19. Some APNs reflected on the provision of end-of-life care during the pandemic and suggested this could be further understood.

No visitor policy - effect on staff, patients and family/ friends. (APN 65)

There were also suggestions to study COVID-19 vaccination, treatment and infection control. Finally, some felt it would be important to study the impact of remote working and the effect that this has on efficiency, costsaving, patient experience and delays in referrals different ways of working.

Patient's experience of virtual nurse-led clinics. (APN 105)

APNs raised several concerns about patient care, not only those with COVID-19 symptoms, but also those unable to access usual services, facing delays to investigations leading to, as one participant put it, 'the second wave'.

Those that are suffering and not being treated as specialities are not seeing patients. There is, I believe, a second wave of deaths coming and it will not be from C-19. (APN 46)

\section{DISCUSSION}

The majority of UK-based APNs in this study reported shortages of staff and PPE during the first 3 months of the coronavirus outbreak. Further, 39\% felt their risk could have been reduced and, worryingly, $21 \%$ felt their employers did not prioritise their safety during this period. Although one respondent did say that they did not blame their employer, they blamed poor national level guidance. These findings mirror the reports from early in the crisis such as Richard Horton's comment in March, in the Lancet on NHS staff lacking PPE and being put at risk. ${ }^{6}$ There were no correlations between primary or secondary care setting or region of the UK and a lack of equipment, staff or concerns about safety, suggesting any issues were national ones rather than regional. As well as increasing the PPE supply, structured, routine testing of healthcare workers will be helpful in managing both future outbreaks and staff health and well-being. ${ }^{21}$

Almost half $(43 \%)$ of APNs surveyed had considered leaving their job over the previous 3 months. For many this meant moving within their current organisations, however, 25\% were considering leaving the nursing profession. This is concerning because APN retention is good, with relatively low turnover. APNs are usually 
very experienced, having worked in the NHS for many years and have previously reported higher levels of job satisfaction and well-being than other areas of the NHS. ${ }^{19}$ Increased fear due to the pandemic has been associated with higher levels of burnout in Spain, ${ }^{22}$ decreased psychological well-being in the USA ${ }^{23}$ and higher intention to leave in the Philippines. ${ }^{24}$

Studies in China and Iran have already shown that healthcare staff who are not properly equipped in this crisis are at high risk of mental health conditions such as depression, anxiety and post-traumatic stress disorder. ${ }^{91325}$ Without extensive, coordinated support and screening for mental health sequelae during the pandemic, ${ }^{26}$ the NHS faces another staffing crisis. Addressing the lack of PPE in some areas and creating a PPE stockpile for future outbreaks would go some way to mitigating this. A recent position paper listed healthcare professionals as a key population of interest for mental health research priorities from the COVID-19 pandemic. ${ }^{27}$

However, there were positive findings as well. Innovative methods of working, being able to spend more time with seriously ill patients and improving their care were all highlighted as examples. Although there have obviously been challenges that must be prevented in the future, there have also been elements that worked well and these can inform a stronger more efficient NHS in the long term.

\section{Limitations}

Due to the time-sensitive nature of the subject matter, this questionnaire was only open for 2 weeks, which may have limited our response rate. This is especially so given that we were targeting health professionals in the middle of a crisis, whose priorities may rightly have been elsewhere.

There are a number of limitations to the survey-only approach. Not least that we could not ask more probing follow-up questions about participant's experiences. However, due to risks of overburdening nurses and the timescales involved, we opted for a survey-only approach.

We only recruited from participants of our existing cohort. While it is the largest of its type that we know about, this approach did not allow for newer APNs to participate, who due to their inexperience in the role, may have offered a different perspective. Also, due to the unregulated nature of APNs in the UK, the cohort allows anyone who self identifies as an APN to join.

\section{CONCLUSION}

During the COVID-19 pandemic, APNs in both primary and secondary care settings across the UK have experienced short staffing and inadequate infection prevention resources. Concerns about the long-term impact of reduced services on patient care have contributed to the distress felt by APNs. The impact of these challenges on the mental health of APNs has significant implications for staff retention across the UK. Half of APNs surveyed were considering a change in job. Examples of innovations that worked well for APNs, such as remote consultations, should be explored in future research from the patient perspective, and where there are shared benefits, carried forward into future nursing practice.

Twitter Emily Wood @emilyfwood

Contributors AR, AT and SR conceptualised the study. All authors contributed to the design of the study and the questionnaire and have read and approved the final manuscript. EW collected data, wrote the primary analysis plan, assisted with the analysis and wrote the paper. RK, SR and BT conducted the qualitative analysis and MS conducted the quantitative analysis. All authors assisted with the interpretation of the results.

Funding The project was funded by the Royal College of Nursing (RCN) as part of the Strategic Research Alliance between the RCN and the University of Sheffield (grant number: SRA861151).

Disclaimer The views expressed are those of the author(s), and not necessarily those of the RCN or University of Sheffield. The RCN aided recruitment to the cohort by sending recruitment emails to their mailing list of credentialed APNs, they had no input into the analysis and interpretation of data, writing the report or decision to publish.

\section{Competing interests None declared.}

Patient consent for publication Not required.

Ethics approval This research was reviewed by the University of Sheffield Research ethics committee and given approval on 3 September 2018 (reference number: 022736). Informed consent was gained from all participants. This research was carried out in accordance with the principles of the Helsinki Declaration. The lead author affirms that the manuscript is an honest, accurate and transparent account of the study being reported.

Provenance and peer review Not commissioned; externally peer reviewed.

Data availability statement Data from this study are be available upon reasonable request to the authors. Individual participant data that underlie the results reported in this article will be available after deidentification (text, tables, figures and appendices). The study protocol will also be available. The data will be accessible beginning 3 months and ending 5 years following article publication to researchers who provide a methodologically sound proposal. Proposals should be directed to e.f.wood@sheffield.ac.uk. To gain access, requestors will be required to sign a data access agreement.

Open access This is an open access article distributed in accordance with the Creative Commons Attribution Non Commercial (CC BY-NC 4.0) license, which permits others to distribute, remix, adapt, build upon this work non-commercially, and license their derivative works on different terms, provided the original work is properly cited, appropriate credit is given, any changes made indicated, and the use is non-commercial. See: http://creativecommons.org/licenses/by-nc/4.0/.

\section{ORCID iDs}

Emily Wood http://orcid.org/0000-0002-1910-6230

Bethany Taylor http://orcid.org/0000-0003-4090-3047

Angela Tod http://orcid.org/0000-0001-6336-3747

\section{REFERENCES}

1 WHO. Naming the coronavirus disease (COVID-19) and the virus that causes it, 2020. Available: https://www.who.int/emergencies/ diseases/novel-coronavirus-2019/technical-guidance/naming-thecoronavirus-disease-(covid-2019)-and-the-virus-that-causes-it [Accessed 22 May 2020].

2 BFPG. COVID-19 Timeline - British Foreign Policy Group, 2020. Available: https://bfpg.co.uk/2020/04/covid-19-timeline/ [Accessed 22 May 2020].

3 Sample I. More than 2M operations cancelled as NHS fights Covid-19. guard, 2020. Available: https://www.theguardian.com/ society/2020/apr/26/more-than-two-million-operations-cancelled-asnhs-fights-covid-19 [Accessed 15 Jun 2020].

4 NHS England. Nhs England "NHS steps up coronavirus fight with two more Nightingale hospitals, 2020. Available: https://www. england.nhs.uk/2020/04/nhs-steps-up-coronavirus-fight-with-twomore-nightingale-hospitals/ [Accessed 22 May 2020]. 
5 NMC. Covid-19 temporary registration, 2020. Available: https://www. nmc.org.uk/news/coronavirus/temporary-registration/ [Accessed 22 May 2020].

6 Horton R. Offline: COVID-19 and the NHS-"a national scandal". Lancet 2020;395:1022.

7 Zhou P, Huang Z, Xiao Y, et al. Protecting Chinese healthcare workers while combating the 2019 novel coronavirus. Infect Control Hosp Epidemiol 2020;41:745-6.

8 Greenberg N, Docherty M, Gnanapragasam S, et al. Managing mental health challenges faced by healthcare workers during covid-19 pandemic. BMJ 2020;368:m1211.

9 Liu Q, Luo D, Haase JE, et al. The experiences of health-care providers during the COVID-19 crisis in China: a qualitative study. Lancet Glob Health 2020;8:e790-8.

10 BPS. Guidance: the psychological needs of health care staff as a result of the coronavirus pandemic. Leicester, 2020. Available: www.ics.ac.uk/ ICS/Education/Wellbeing/ICS/Wellbeing.aspx [Accessed 12 Jun 2020].

11 Herron JBT, Hay-David AGC, Gilliam AD, et al. Personal protective equipment and Covid 19- a risk to healthcare staff? Br J Oral Maxillofac Surg 2020;58:500-2.

$12 \mathrm{RCN}$. Second personal protective equipment survey of UK nursing staff report. London, England, 2020

13 Zhang SX, Liu J, Afshar Jahanshahi A. At the height of the storm: Healthcare staff's health conditions and job satisfaction and their associated predictors during the epidemic peak of COVID-19. Brain Behav Immun 2020

14 Schwartz J, King C-C, Yen M-Y. Protecting Healthcare Workers During the Coronavirus Disease 2019 (COVID-19) Outbreak: Lessons From Taiwan's Severe Acute Respiratory Syndrome Response. Clin Infect Dis 2020 https://academic.oup.com/cid/advance-article/doi/ (accessed June 12, 2020).

15 Richards M, Anderson M, Carter P, et al. The impact of the COVID-19 pandemic on cancer care. Nat Cancer 2020;1:565-7.

16 International Council of Nurses. The scope of practice, standards and competencies of the advanced practice nurse. Geneva: ICN regulatory series, 2008.

17 Health Education England. Multi-professional framework for advanced clinical practice in England 2017.
18 Ford M. International guidelines launched on role of advanced nurse | nursing times. Nurs. times, 2020. Available: https:// www.nursingtimes.net/news/workforce/international-guidelineslaunched-on-role-of-advanced-nurse-17-04-2020/ [Accessed 22 May 2020].

19 Wood E, King R, Robertson S, et al. Advanced practice nurses experiences and well-being: baseline demographics from a cohort study. J Nurs Manag 2020;28:959-67.

20 Srivastava A, Thomson SB. Framework analysis: a qualitative methodology for applied policy research. J Adm Gov 2009:72 https:// papers.ssrn.com/sol3/papers.cfm?abstract_id=2760705 (accessed July 14, 2017).

21 Bielicki JA, Duval X, Gobat N. Monitoring approaches for health-care workers during the COVID-19 pandemic. Lancet Infect Dis 2020;0.

22 Soto-Rubio A, Giménez-Espert MdelC, Prado-Gascó V. Effect of Emotional Intelligence and Psychosocial Risks on Burnout, Job Satisfaction, and Nurses' Health during the COVID-19 Pandemic. Int $J$ Environ Res Public Health 2020;17:7998.

23 Sagherian K, Steege LM, Cobb SJ, et al. Insomnia, fatigue and psychosocial well-being during COVID-19 pandemic: a crosssectional survey of hospital nursing staff in the United States. J Clin Nurs 2020. doi:10.1111/jocn.15566. [Epub ahead of print: 20 Nov 2020].

24 De los Santos J, Labrague L. Impact of COVID-19 on the psychological well-being and turnover intentions of frontline nurses in the community: a cross-sectional study in the Philippines. medRxiv Prepr 2020

25 Fernandez R, Lord H, Halcomb E, et al. Implications for COVID-19: a systematic review of nurses' experiences of working in acute care hospital settings during a respiratory pandemic. Int J Nurs Stud 2020;111:103637.

26 Preti E, Di Mattei V, Perego G, et al. The psychological impact of epidemic and pandemic outbreaks on healthcare workers: rapid review of the evidence. Curr Psychiatry Rep 2020;22:1-22.

27 Holmes EA, O'Connor RC, Perry VH, et al. Multidisciplinary research priorities for the COVID-19 pandemic: a call for action for mental health science. Lancet Psychiatry 2020;7:547-60. 